\title{
Climatology of the cusp-related thermospheric mass density anomaly, as derived from CHAMP observations
}

\author{
S. Rentz and H. Lühr \\ Helmholtz-Centre Potsdam, GFZ German Research Centre for Geosciences, Potsdam, Germany \\ Received: 28 February 2008 - Revised: 31 July 2008 - Accepted: 11 August 2008 - Published: 18 September 2008
}

\begin{abstract}
We report on the thermospheric mass density anomaly in the vicinity of the ionospheric cusp. A systematic survey of the anomalies is presented, based on a statistical analysis of 4 years of data (2002-2005) obtained by the accelerometer onboard CHAMP. The anomalies are detected during all years and seasons in both hemispheres but with stronger signatures in the Northern Hemisphere. For the same geophysical conditions, solar flux and geomagnetic activity the anomalies in the north are larger by a factor of about 1.35. Over the course of the survey period the amplitude decreases by more than a factor of 5 while the level of solar flux reduces by a factor of 2 . The anomaly strength also depends on the solar wind input. The merging electric field, $E_{\mathrm{merg}}$, is generally enhanced for about an hour before the anomaly detection. There is a quadratic response of the anomaly amplitude to $E_{\text {merg }}$. For geophysical conditions of P10.7 $<150$ and $E_{\text {merg }}<1 \mathrm{mV} / \mathrm{m}$ hardly any events are detected. Their amplitudes are found to be controlled by an additive effect of P10.7 and $E_{\text {merg }}$, where the weight of $E_{\mathrm{merg}}$, in $\mathrm{mV} / \mathrm{m}$, is by about 50 times higher than that of the solar flux level. The solar zenith angle and the influence of particle precipitation are found to play a minor role as a controlling parameter of seasonal variation. The well-known annual variation of the thermospheric density with a minimum around June also influences the formation of the cusp anomalies. This leads to a clear hemispheric asymmetry with very weak anomalies in the south during June solstice, which is supposed to be a combined effect of the minimum in annual variation and the seasonal decrease of solar insolation in this region.
\end{abstract}

Keywords. Ionosphere (Ionosphere-magnetosphere interactions) - Meteorology and atmospheric dynamics (Thermospheric dynamics) - Radio science (Ionospheric physics)

Correspondence to: S. Rentz

(stefanie@gfz-potsdam.de)

\section{Introduction}

The polar upper atmosphere in the altitude range 100$1000 \mathrm{~km}$ is affected by many different processes. The influence of solar extreme ultraviolet (EUV) radiation, the Earth's magnetic field, magnetospheric particle precipitation, plasmadynamic and hydrodynamic forces are interacting in this region. This part of the atmosphere, the thermosphere, is modified by variations in solar and geomagnetic activity, as well as by seasons or local characteristics of particle flux, electric field variation, ion drift, neutral gas flow and atmospheric dynamics.

Indeed, the parameters of this complex system have been studied since the beginning of spectroscopic measurements. The idea that the upper atmosphere is disturbed and heated by solar particles was first suggested in the 1930s (e.g. Appleton and Ingram, 1935). Heating, dissociation, and ionization in the upper atmosphere were referred to solar UV radiation (Mitra, 1947). Since solar UV radiation was the only energy input to the thermosphere in the early static diffusion models (Nicolet, 1960), first empirical thermospheric models followed this concept. In the late 1950s, Jacchia first documented solar and geomagnetic energy effects from observations of 1958 Delta One and 1958 Beta Two satellites (Jacchia, 1959). In 1963, Jacchia and Slowey detected particle energy flow into the high latitude thermosphere during geomagnetic storms. Besides the work of Jacchia (1961), Pätzold's model (Pätzold, 1963) is one of the first that contains a contribution to a density enhancement by geomagnetic heating. In 1964 , a $K_{p}$ - or $A_{p}$-dependent exospheric temperature was included in the Jacchia model (Jacchia, 1964), and it was first reported on an anomalously large density increase in the polar region that was exceeding the expected effects at low latitudes by about 4 to 5 times (Jacchia and Slowey, 1964). Simultaneously, the first polar orbiting satellites in operation allowed inferring the density enhancements from orbital parameter analysis (Jacobs, 1967).

Published by Copernicus Publications on behalf of the European Geosciences Union. 
First reports on particle fluxes in the cusp region date to 1971: Heikkila and Winningham (1971) refer to observations at low altitudes with the ISIS satellite, while Frank (1971) and Russell et al. (1971) account for high-altitude cusp observations with IMP-5 and OGO-5, respectively. They reported about direct observations of large fluxes of relatively low energy charged particles $(\sim 1 \mathrm{keV})$ which are precipitating continuously into the atmosphere through the magnetic field region at the magnetopause where the magnetic field lines diverge. With the help of Alouette and ISIS satellite data the influence of charged particle input during quiet times was studied and the average particle precipitation region could be localized (Olson, 1972). It was found to be best described in solar geomagnetic coordinates rather than in geographic coordinates. Based on data from Spades and Logacs satellites (Bruce, 1973; Moe et al., 1977), a global thermospheric density model was developed by Moe and Moe (1975). It takes account of the density bulge caused by energy deposition through the cusp. Between fall 1981 and spring 1983, DE-2 satellite data revealed an enhanced electron temperature in the dayside polar upper atmosphere. Its position depends mainly on the level of auroral geomagnetic activity (AE index) rather than on the $B_{z}$ component of the interplanetary magnetic field (Prölss, 2006).

The development of incoherent scatter radar techniques and their installation in auroral regions, such as EISCAT, revealed new possibilities for ground-based studies of the upper atmosphere, especially of the ionised component. Whilst this component has been subject of numerous scientific studies for a long time (e.g. Lathuillère and Brekke, 1985; Stubbe, 1996; Yordanova et al., 2007) the investigation of the neutral component was often restrained by the lack of suitable measurement methods. It gained attention primary during recent years (e.g. Bruinsma et al., 2004; Liu et al., 2005; Sutton et al., 2005; Menvielle et al., 2007).

The Earth observation satellite CHAMP (CHAllenging Minisatellite Payload) contributes significantly to these investigations (Reigber et al., 2002). CHAMP is orbiting within this complex system of the upper atmosphere at $\sim 400 \mathrm{~km}$ altitude. The onboard high-sensitive tri-axial accelerometer allows for the first time continuous, physically clean and high resolution measurements of the neutral gas component with good global and spatial coverage (Bruinsma et al., 2004; Liu et al., 2005) for both, the Northern and Southern Hemisphere. From these data, we can derive the total mass density as well as information about thermospheric neutral winds (Liu et al., 2006; Lühr et al., 2007). Liu et al. (2005) found that the air density at polar regions increases with increasing geomagnetic activity. The diurnal density variation dominates the total mass density distribution, but a cusp-related density enhancement is visible, even during quiet conditions (Liu et al., 2005). In a case study of 25 September 2000, Lühr et al. (2004) showed that the air drag measured along the CHAMP orbit sometimes contains superimposed small-scale features, which can reach almost a factor of 2 above the ambient density under solar maximum conditions. These drag peaks coincide well with cusp crossings. A continuous occurrence was supposed. The cusp is the region where magnetosheath plasma can enter into lower altitudes most directly (Russell, 2000). According to Lühr et al. (2004), these incoming particles are supposed to be associated with field-aligned currents (FACs). These currents may fuel local cusp heating, which can be responsible for air up-welling, leading to density enhancements at higher altitudes. Lühr et al. (2004) suggested that in particular the simultaneously observed intense small-scale FACs may play an important role. They provide a strong coupling of the carried Alfvén wave with the high-latitude ionosphere, which means, magnetospheric energy is dissipated most efficiently in the atmosphere at ionospheric heights (Vogt, 2002).

Schlegel et al. (2005) were the first who combined CHAMP data with EISCAT measurements to investigate the density anomalies at cusp latitudes. During a sevenday campaign in February 2002, they frequently detected density maxima in the vicinity of the cusp with spatial scales of $100 \mathrm{~km}$ to $2000 \mathrm{~km}$ and with amplitudes of up to $50 \%$ above the ambient density. Only recently these local phenomena gained interest in the modelling community. Demars and Schunk (2007) succeeded in reproducing the CHAMP-observed density enhancements in the cusp with their high-resolution thermospheric model. According to their results, Joule heating in the cusp generates vertical transport which causes a neutral fountain effect. Hence, the neutral density is boosted up to higher altitudes and subsequently diverted into poleward and equatorward directions. In their model, Demars and Schunk (2007) had to gear up the heating in the E-layer by a factor of 110 to obtain a cusp density bulge as reported by Lühr et al. (2004).

The detailed reports on cusp air density enhancements are limited so far to event studies which may be regarded as a valuable tool for identifying relevant heating mechanisms. We extend the work of event studies by considering a larger number of cases. The identification of the role of the various possible contributors to the air density enhancement (like solar EUV radiation, magnetic activity or atmospheric composition changes) requires a longer observational period. In this study we want to investigate the detailed characteristics of the mass density anomalies in the cusp region. In particular, the dependence on possible solar and/or magnetospheric drivers will be studied. Furthermore, we want to find out the specific thermospheric conditions or seasons which may favour the formation of cusp density bulges. Our approach for making progress in the investigation of the cusp density anomaly is to take advantage of 4 years of continuous CHAMP air drag measurements. Within this period, the above mentioned controlling parameters vary considerably and a correlation analysis is expected to reveal significant results.

In the section to follow we will first describe the considered data set and then outline the analysis approach. 
Subsequently, we present the statistical properties of the density anomaly and perform a correlation analysis with a selection of the possible controlling parameters. In the Discussion section we make an attempt to interpret the obtained results. Finally, the major conclusions are summarised.

\section{Observations and data analysis}

\subsection{CHAMP dataset and density anomaly identification}

The CHAMP satellite, launched on 15 July 2000, cycles around the Earth on a near-polar (inclination $87.25^{\circ}$ ) and almost circular orbit at an altitude of about $400 \mathrm{~km}$ (Reigber et al., 2002). Therewith, the satellite provides an excellent latitudinal coverage, even in polar regions. It takes eleven days for the orbital plane to pass through one hour of local time, thus CHAMP covers all local times once in 131 days. From its original altitude of $456 \mathrm{~km}$ the spacecraft decayed to about $425 \mathrm{~km}$ at the beginning of our observation period and to barely $360 \mathrm{~km}$ at the end of 2005 . Hence, the investigations are focusing on an average altitude of $400 \mathrm{~km}$.

We are analyzing STAR (Space Three-axis Accelerometer for Research missions) accelerometer data, taken onboard CHAMP during the period 1 January 2002 to 31 December 2005. From the selected interval a total of 22734 passes over the northern polar area and 22668 passes over the southern polar area are considered. The dataset consists of preprocessed Level-2-data of the non-gravitational accelerations acting on the spacecraft. Spurious spikes and accelerations, which are caused by attitude manoeuvres, are removed, and the data are averaged over a period of $10 \mathrm{~s}$. This is equivalent to a sampling distance of $76 \mathrm{~km}$. Additionally, the acceleration, which is caused by solar radiation pressure, is subtracted from the readings. Readings from very active periods $\left(D_{s t}<-200\right)$ are removed. The acceleration (deceleration), $\boldsymbol{a}$, due to air drag can be expressed as

$$
\begin{aligned}
\boldsymbol{a} & =\frac{1}{2} \rho \frac{C_{D}}{m} A_{\mathrm{eff}} V^{2} \hat{\boldsymbol{v}}, \\
\rho & =\frac{2 m \boldsymbol{a} \cdot \hat{\boldsymbol{v}}}{C_{D} A_{\mathrm{eff}} V^{2}},
\end{aligned}
$$

where $\rho$ is the local thermospheric mass density, $C_{D}$ the drag coefficient, $m$ the spacecraft mass, $V$ the spacecraft velocity relative to the ambient air, $\hat{v}$ the unit vector of the velocity in ram direction, $A_{\text {eff }}=A_{x} \cos \alpha+A_{y} \sin |\alpha|$ is the effective cross-sectional area with $\tan \alpha=\frac{a_{y}}{a_{x}}$, and $V^{2}=v_{\text {orbit }}{ }^{2}+v_{\text {cross }}{ }^{2}$ with contributions from the orbital velocity, $v_{\text {orbit }}$, and crosstrack wind velocity, $v_{\text {cross. }}$. The measured acceleration components $a_{x}$ and $a_{y}$ are pointing in flight direction and cross-track, respectively. From Eq. (2) we see that the acceleration vector is aligned with the velocity, $\hat{\boldsymbol{v}}$. Thus we can relate the component ratios of these two vectors: $v_{\text {cross }} / v_{\text {orbit }}=-a_{y} / a_{x}$. Since CHAMP is polar-orbiting, $v_{\text {cross }}$ is the cross-track wind and $v_{\text {orbit }}$ the spacecraft mean velocity along the orbit. It amounts to $7.6 \mathrm{~km} / \mathrm{s}$. Thus, it is possible to take into account the influence of cross-track winds when calculating the density values. The effect of head and tail winds is not considered. We assume that they average out over a four-year period. Furthermore, Lühr et al. (2007) showed that the wind velocities in the vicinity of the cusp are fairly low.

The high-sensitive accelerometer offers a resolution of more than $3 \times 10^{-9} \mathrm{~m} / \mathrm{s}^{2}$. For typical CHAMP characteristics, $m=500 \mathrm{~kg}, A_{\mathrm{eff}}=0.8 \mathrm{~m}^{2}$, and $C_{D}=2.2$ this converts to a mass density resolution of $6 \times 10^{-14} \mathrm{~kg} / \mathrm{m}^{3}$. For further details on the treatment of the accelerometer data and their uncertainty the reader is refered to the Appendix of Liu et al. (2006). Since we are primarily interested in the cusp density enhancement, possible biases in some of the satellite parameters are not important here.

For some of the interpretations the density measurements have been normalized to a common altitude of $400 \mathrm{~km}$ via the relation

$\rho(400 \mathrm{~km})=\rho(h) \frac{\rho_{\text {MSIS }}(400 \mathrm{~km})}{\rho_{\text {MSIS }}(h)}$,

where $h$ is the actual height of CHAMP above the ellipsoid. The model densities are taken from the NRLMSISE00 atmospheric model (Picone et al., 2002). Since the CHAMP orbit altitude varies roughly within one scale height $(H \approx 60 \mathrm{~km})$, errors caused by the normalisation are expected to be small: Liu et al. (2007) quoted an uncertainty of 5\%. This is regarded acceptable, when comparing a multi-year period over which the spacecraft decays by about $65 \mathrm{~km}$. The height-normalised densities are used for the Figs. 4 and 5, while the Figs. 6 to 10 have been drawn for density values at CHAMP orbital height. Respective plots derived from height-normalised densities (not shown) reveal very similar results.

In order to focus on the density anomaly at cusp latitudes, we removed large-scale features, such as the diurnal and/or latitudinal variation from the records. This is done by subtracting the corresponding NRLMSISE-00 density, and in addition a remaining linear bias between model and observation is removed between $55^{\circ}$ and $89^{\circ}$ corrected geomagnetic (cgm) latitude. Figure 1 illustrates the background removal approach for one cusp crossing of both hemispheres. The CHAMP density, $\rho$, and the corresponding NRLMSISE-00 density, $\rho_{\text {MSIS }}$, are derived for the same location and point in time. MSIS does not reproduce local features at high latitudes well, and therefore it is appropriate to separate the large-scale background features from the detailed CHAMP density readings. As expected, a latitude dependent trend is left in the observations along an overpass, which is regarded as a so-called linear bias. The value of the bias is derived from a linear interpolation between the density differences at $55^{\circ} \mathrm{cgm}$ latitude and the highest point reached in cgm latitude. In Fig. 1 this linear trend is displayed in grey. It was 

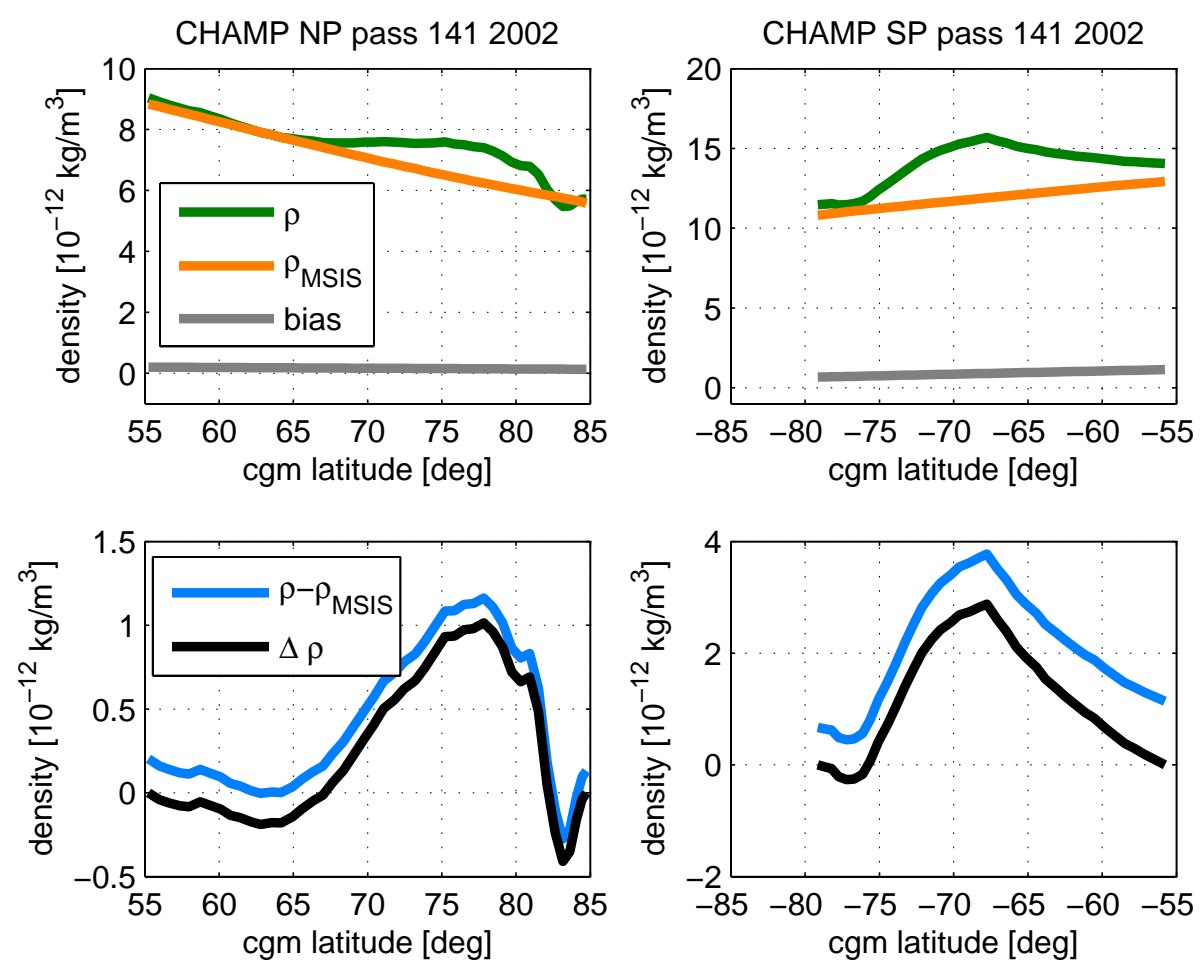

Fig. 1. Schematic overview of the anomaly identification procedure for the example of the overpass 141/2002 (10 January 2002). The left panels refer to the Northern Hemisphere and the right panels to the southern hemispheric part of the pass. The upper panels display the density from CHAMP data (green), the corresponding model density from NRLMSISE-00 (orange), and the linear bias function (grey). In the lower panels, the blue line indicates the difference between CHAMP-measured and NRLMSISE-00 density. The black line denotes the final density anomaly, $\Delta \rho$, which is used in this study.

also subtracted from the density data. Hence, the density anomaly, $\Delta \rho$, was calculated: $\Delta \rho=\rho-\rho_{\text {MSIS }}-\rho_{\text {bias }}$. Here, $\rho$ is the density derived from the accelerometer readings according to Eq. (2), $\rho_{\text {MSIS }}$ is the MSIS model density for the location and time of $\rho$ and $\rho_{\text {bias }}$ is the corresponding density value from the linear bias function. Therewith, we are able to diminish the influence of large-scale trends or biases between model and measurements on our mass density anomaly, $\Delta \rho$.

Furthermore, we calculated the relative density anomaly, $d \rho_{\text {rel }}$ as:

$d \rho_{\text {rel }}=\frac{\rho}{\rho-\Delta \rho}$,

The relative density deviations may help to find the dependence of the size of the anomaly on the ambient mass density.

\subsection{Considered parameters}

Our aim is to determine the dependence of the cusp-related density anomaly on various parameters which are briefly described in the following.

The solar flux variation is approximated by the F10.7 and P10.7 indices, which reflect the strength of the EUV radia- tion. According to Guo et al. (2007) a use of multiple solar irradiance indices, in fact a combination of F10.7, $S_{\mathrm{EUV}}(30-$ $120 \mathrm{~nm})$ and $S_{\mathrm{FUV}}(120-130 \mathrm{~nm})$ with a time lag of 1 day provides a higher correlation than using exclusively F10.7. Similarly good results can be obtained when applying the P10.7 index from the previous day, which is calculated as:

$\mathrm{P} 10.7=\frac{1}{2}\left(\mathrm{~F} 10.7+\overline{\mathrm{F} 10.7_{81 \text { days }}}\right)$,

(Richards et al., 1994). Previous studies have shown that P10.7 is more appropriate as a linear indicator also for the ionospheric response to solar EUV radiation (L. Liu et al., 2006). As can be seen from their Fig. 1, P10.7 gives smoother solar flux variations than F10.7 which is more emphasizing the shorter activity phases. Since we are interested in the climatological behaviour, we prefer P107. The F10.7 values are downloaded from the SPIDR website http:/spidr.ngdc.noaa.gov/.

The dependence on the solar irradiation can be sufficiently described by the solar zenith angle (SZA). It is the sun's angular distance from the vertical and depends on season and local time. It can be calculated as:

$\cos (\mathrm{SZA})=\sin \beta \sin \delta+\cos \beta \cos \delta \cos t$ 
where $\beta$ is the geographic latitude, $\delta$ is the solar declination, and $t$ is the local time as angle. For $\mathrm{SZA}>100^{\circ}$ the ionospheric $\mathrm{E}$ region is in darkness, for $\mathrm{SZA} \leqq 100^{\circ}$ it is sunlit (Schlegel, 1988). The SZA is used in this study for estimating the ionospheric conductivity.

An important quantity controlling the solar windmagnetosphere coupling processes is the interplanetary magnetic field (IMF). The IMF components $B_{y}$ and $B_{z}$, which are considered in this study, are derived from the 1-min final data of the Advanced Composition Explorer (ACE) satellite, published in the NASA Science Center. The transit time of each reading from the ACE location at $L 1$ point to the magnetopause was computed individually using the actual solar wind speed data. Following Vennerstrøm et al. (2002) an additional time delay of $15 \mathrm{~min}$ was added to take account of the distance between the magnetopause and the polar ionosphere. The merging electric field, $E_{\mathrm{merg}}$, has been derived from merging theory assuming that there is an equal magnitude of the electric field in the solar wind, the magnetosheath and on the magnetospheric sides of the magnetopause (Kan and Lee, 1979):

$E_{m}=v_{S W} \sqrt{B_{y}{ }^{2}+B_{z}^{2}} \sin ^{2}\left(\frac{\theta}{2}\right)$

where $B_{y}$ and $B_{z}$ are the IMF components, $v_{S W}$ is the solar wind speed, and $\theta$ the IMF clock angle in GSM coordinates. In a superposed epoch analysis Ritter et al. (2004) found the merging electric field to be a suitable geoeffective solar wind parameter. Therefore, in our study the merging electric field will be used as a measure for the solar wind input to the magnetosphere. It has been found in previous studies that the tilt angle of the geomagnetic dipole with respect to the GSM $y-z$ plane has an influence on the location of the cusp (e.g. Zhou et al., 1999). We will investigate how the location of the density anomalies depends on the tilt angle.

A further mechanism that is modifying the ionospheric conductivity at auroral latitudes is particle precipitation. Since we do not include particle measurements in this study, we provide an estimate of this effet in the cusp region based on modelling results of Millward et al. (1999) in Sect. 4.2.

\section{Statistical analysis}

We make use of 4 years of CHAMP data (1 January 200231 December 2005) to investigate the statistical features of the mass density anomaly in the dayside high-latitude thermosphere. The data were sorted by cgm coordinates using the APEX system (Richmond, 1995) in order to emphasize the magnetospheric input. Since we are interested in daytime features, we picked all measurements taken between 08:00 and 16:00 magnetic local time (MLT). Supposing the cusp to be positioned at auroral latitudes, we select a range of $55 \ldots 89^{\circ}\left(-55 \ldots-89^{\circ}\right) \mathrm{cgm}$ latitude in the Northern (Southern) Hemisphere. Chosing this segment of latitude and local time we intended to cover the cusp location and the surrounding area since we wanted to capture the whole density anomaly distribution which is not expected to be limited to the magnetospheric cusp proper.

For a binning of the data the polar region is divided into 17 concentric latitude rings (covering $24 \mathrm{~h}$ of magnetic local time) with a width of $2^{\circ} \mathrm{cgm}$ latitude each, starting with ring $1\left(89 \ldots 87^{\circ}\left(-89 \ldots-87^{\circ}\right) \mathrm{cgm}\right.$ latitude) to ring $17\left(57 \ldots 55^{\circ}\left(-57 \ldots-55^{\circ}\right) \mathrm{cgm}\right.$ latitude). Subsequently, these rings are subdivided into equal-area bins with a range of about $222 \times 232 \mathrm{~km}$. Therefore, we divide the (innermost) ring 1 into 6 sectors, ring 2 into $2 \times 6$ sectors ... ring 17 into $17 \times 6$ sectors. The binning procedure is equivalent to the one applied in Lühr et al. (2007). All available density value samples, $\rho$, from a pass over a bin are dropped into the appropriate bin. For this study, we consider only the sector of $\pm 4 \mathrm{~h}$ around noon.

The large number of passes ensures many entries in each bin, ranging from $>8000$ near the pole to at least 500 at $55^{\circ} \mathrm{cgm}$ latitude. The sample distribution is shown in Fig. 2, where all available overflights have been combined. We inserted the magnetospheric cusp position as obtained by Newell and Meng (1992) in black. For average conditions it ranges from $\approx 10: 25 \mathrm{MLT}-13: 30 \mathrm{MLT}$ and $78^{\circ}-79^{\circ} \mathrm{cgm}$ latitude. Figure 3 shows the distribution of anomaly detection numbers for the different P10.7 levels, separately for the two hemispheres. If the $\Delta \rho$ maximum of an overpass was detected between $\left|60^{\circ}\right|$ and $\left|80^{\circ}\right| \mathrm{cgm}$ latitude and 08:00 and 16:00 MLT we regard it as an anomaly event. We use P10.7 values with a time lag of 1 day, as recommended by Guo et al. (2007). Most density peaks occur during phases of low solar activity (P10.7 $\leq 130)$ with highest occurrence numbers within the interval $95 \leq \mathrm{P} 10.7 \leq 105$. There are rather few events in the interval around $P 10.7=140$. This local minimum can be regarded as a demarcation between high and low solar fluxes. The occurrence number in the last bar is an accumulation of all events with $\mathrm{P} 10.7 \geq 215$. The distribution is almost the same in both hemispheres. All together, we have detected 13787 (13051) density anomalies in the Northern (Southern) Hemisphere.

\subsection{Cusp-related density enhancement}

In the following we are going to subdivide the entries in each bin according to certain criteria in order to find out dependences of the density anomaly on ambient conditions.

Figure 4 presents the observed density anomaly distribution, $\Delta \rho$ (mean values, normalised to $400 \mathrm{~km}$ altitude) in the dayside polar cusp separately for the years 2002-2005. The upper row displays the northern hemispheric situation; the lower row refers to the southern hemispheric distribution. Concentric rings mark the magnetic latitudes $60^{\circ}, 70^{\circ}, 80^{\circ}$. The numbers outside the circle flag the magnetic local time. We present density values, $\Delta \rho$, in the 08:00-16:00 MLT sector between $89 \ldots 55^{\circ}\left(-89 \ldots-55^{\circ}\right) \mathrm{cgm}$ latitude for the 
$\mathrm{NH}$
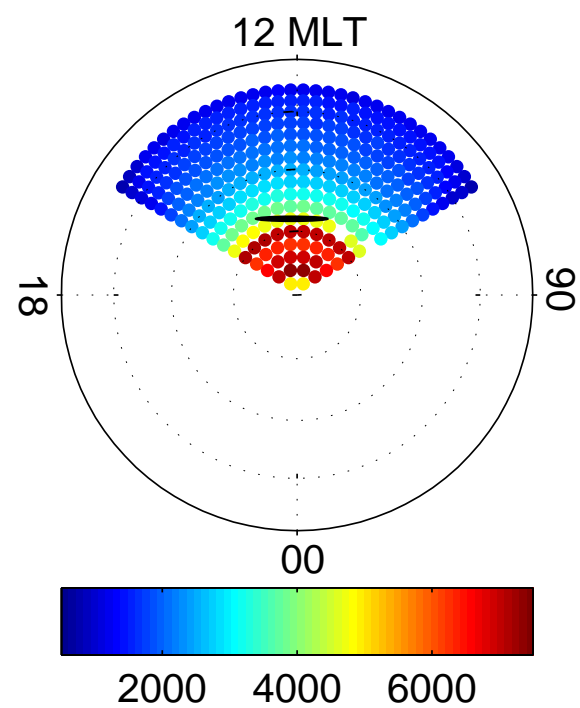

$\mathrm{SiH}$
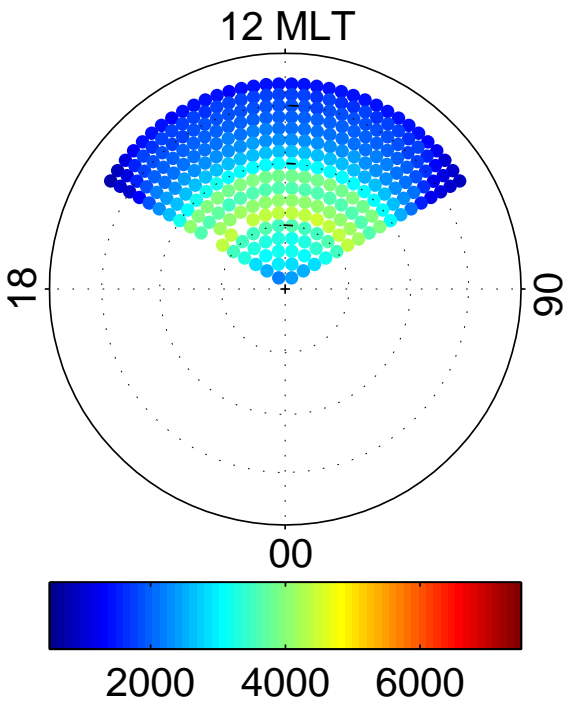

Fig. 2. Sample number per bin, distribution from all CHAMP passes 2002-2005 over the dayside Northern (left) and Southern (right) Hemisphere. The magnetospheric cusp location quoted by Newell and Meng (1992) was inserted in black.
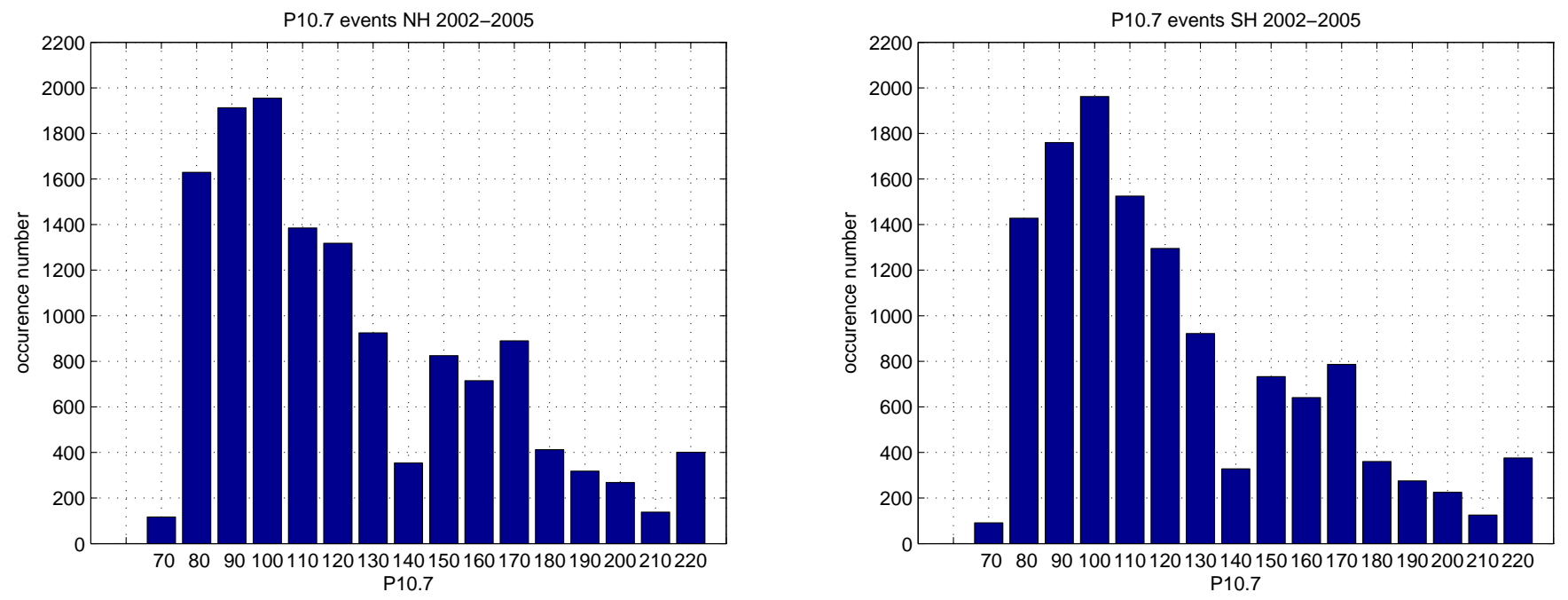

Fig. 3. Occurrence distribution of density anomaly detections at different P10.7 levels. In the bar P10.7=220 all events from higher flux levels are accumulated.

Northern (Southern) Hemisphere. Due to the large change of the density anomaly strength with time, we use different scales for the years.

The density enhancement is visible in each frame. At northern cusp latitudes, it is most intensive in 2002 with average density anomalies up to $1.43 \times 10^{-12} \mathrm{~kg} / \mathrm{m}^{3}$. However, it is decreasing by a factor of almost 6 until 2005. The latitudinal spread of the area of large density anomalies decreases continuously from 2002 to 2005 . Considering the full latitudinal width at half maximum (FWHM, cf. Table 1) of the mean density anomaly distribution as a characteristic number, it reduces from $13.8^{\circ}$ in 2002 to $12.9^{\circ}$ in 2005 in the Northern Hemisphere. However, the latitude of the average peak is found to be between $72^{\circ}$ and $74^{\circ}$. We obtained the peak density, the cgm latitude and FWHM by averaging the values of each latitude ring and then plotted the profile across all rings. The resulting curve is used to identify the average amplitude and latitude of the anomaly, as 
Table 1. Peak and standard deviation (std) of the longitudinally averaged amplitude, its cgm latitude, full latitudinal width at half maximum (FWHM) and the magnetic local time (MLT) of the density anomaly peak in the Northern (NH) and Southern (SH) Hemisphere for the different years and seasons. The standard deviation and the mean error of the average of the FWHM amount to about $10^{\circ}$ and $2.43^{\circ}$ in latitude in all cases, respectively.

\begin{tabular}{lccccc|ccccc}
\hline $\begin{array}{l}\text { time } \\
\text { period }\end{array}$ & $\begin{array}{c}\text { max } \\
\text { of } \Delta \rho \\
{\left[10^{-12} \mathrm{~kg} / \mathrm{m}^{3}\right]}\end{array}$ & $\begin{array}{c}\text { Nem } \\
\text { cgm } \\
{\left[^{\circ}\right] \pm 1^{\circ}}\end{array}$ & $\begin{array}{c}\text { FWHM } \\
{\left[{ }^{\circ}\right]}\end{array}$ & $\begin{array}{c}\text { MLT } \\
\pm 15 \text { min } \\
{[\mathrm{h}]}\end{array}$ & $\begin{array}{c}\text { max } \\
\text { of } \Delta \rho \\
{\left[10^{-12} \mathrm{~kg} / \mathrm{m}^{3}\right]}\end{array}$ & $\begin{array}{c}\text { Std } \\
\text { cgm } \\
{\left[{ }^{\circ}\right] \pm 1^{\circ}}\end{array}$ & $\begin{array}{c}\text { FWHM } \\
{\left[{ }^{\circ}\right]}\end{array}$ & $\begin{array}{c}\text { MLT } \\
\pm 15 \text { min } \\
{[\mathrm{h}]}\end{array}$ \\
\hline 2002 & 1.43 & 1.75 & 74 & 13.8 & $11: 45$ & 1.33 & 9.01 & -68 & 16.7 & $11: 05$ \\
2003 & 0.83 & 1.16 & 74 & 13.6 & $12: 15$ & 0.58 & 0.95 & -70 & 14.6 & $12: 13$ \\
2004 & 0.37 & 0.67 & 72 & 12.8 & $12: 40$ & 0.25 & 0.59 & -76 & 16.1 & $12: 17$ \\
2005 & 0.24 & 0.49 & 72 & 12.9 & $13: 07$ & 0.14 & 0.44 & -76 & 13.0 & $11: 09$ \\
\hline ME & 0.69 & 1.07 & 74 & 12.3 & $11: 15$ & 0.42 & 0.84 & -76 & 12.7 & $12: 17$ \\
JS & 0.70 & 0.97 & 72 & 15.9 & $12: 13$ & 0.71 & 8.23 & - & - & - \\
SE & 0.85 & 1.16 & 72 & 14.0 & $13: 33$ & 0.64 & 1.44 & -68 & 16.8 & $11: 05$ \\
DS & 0.83 & 1.50 & 74 & 12.8 & $11: 45$ & 1.14 & 8.41 & -68 & 15.2 & $08: 54$ \\
\hline
\end{tabular}
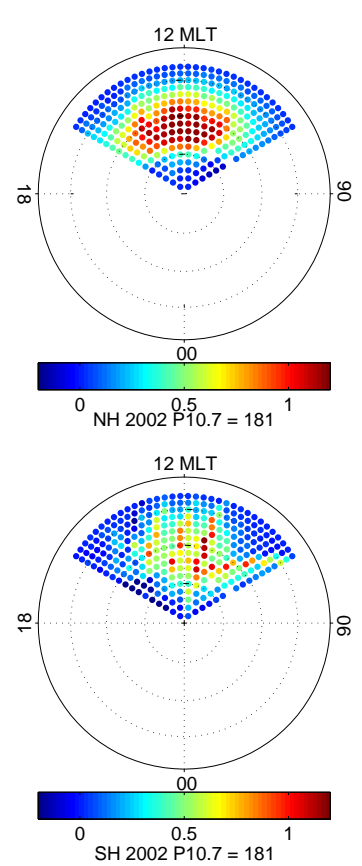
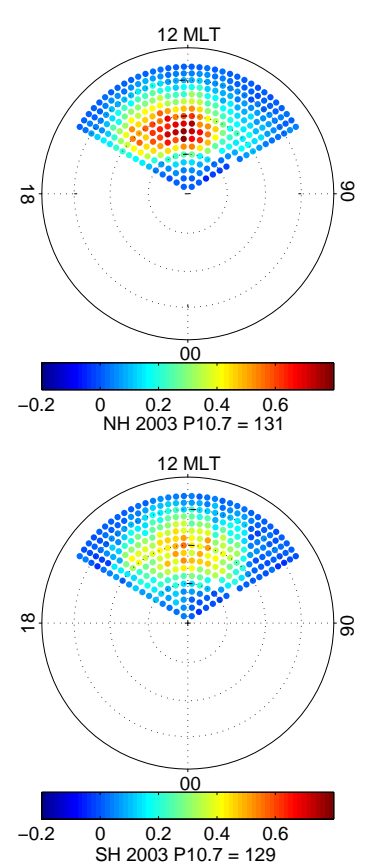
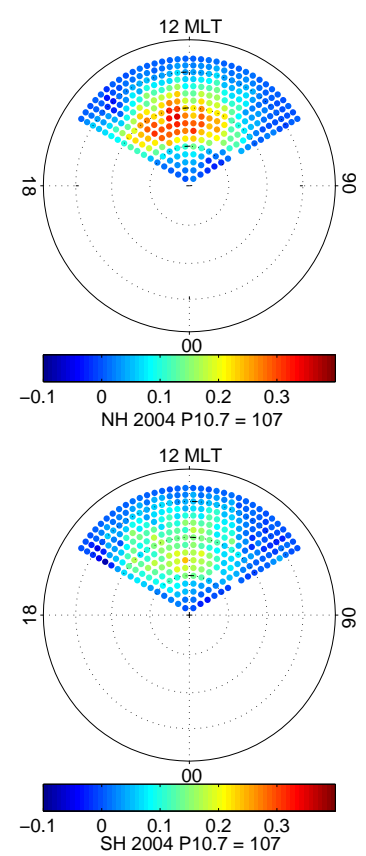

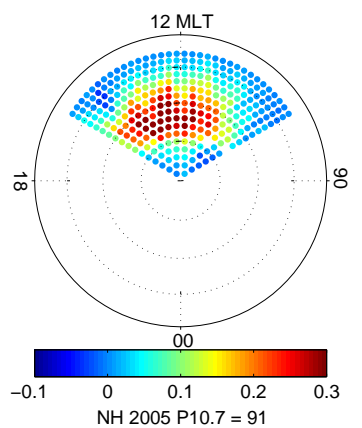

$12 \mathrm{MLT}$

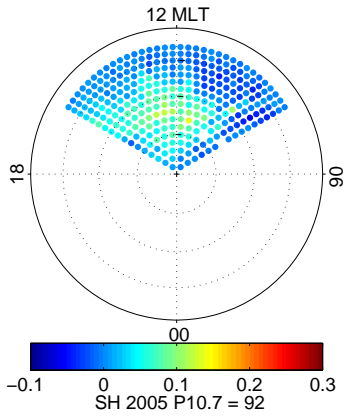

Fig. 4. Statistical survey over four years of the density anomaly amplitude (in $10^{-12} \mathrm{~kg} / \mathrm{m}^{3}$ ) within the dayside polar region of the Northern (upper row) and the Southern (lower row) Hemisphere. Note the different scales. Yearly averaged P10.7 values are listed below the plots.

well as for calculating the FWHM. This method (of FWHM derivation) is not straightforward. It requires a Gaussian distribution of the averaged latitudes. That is why we applied the Wilcoxon rank sum test to evaluate the statistical significance of changes in FWHM. The probability of observing equal medians between the given profile and the corresponding Gaussian distribution at an uncertainty level of $5 \%$ is $>0.89$ except for SH JS. The centre MLT of the density peak moves towards the afternoon with the years. At $74^{\circ} \mathrm{cgm}$ latitude the bin size is $30 \mathrm{~min}$ in MLT. As can be seen in Table 1, it appears $\approx 80 \mathrm{~min}$ later in 2005 than in 2002. This may, however, be an effect of the diminishing anomaly amplitude in the later years so that other auroral processes can have comparable influences on the thermosphere.

For comparison, we decided to apply the same scale in Fig. 4 for the Northern and Southern Hemispheres. This scale was chosen to fit the Northern Hemisphere observations, where the phenomenon is more distinct. In the southern polar region the density anomaly is less convincing. Although it can be identified in each year, its structure does 
not hold any common features. With density values up to $0.58 \times 10^{-12} \mathrm{~kg} / \mathrm{m}^{3}$ it is maximal in $2003(\approx 70 \%$ of the northern hemispheric 2003 maximum density). The anomaly seems to be shifted to the morning hours compared to the northern hemispheric density distribution.

In the Southern Hemisphere, the FWHM is largest in 2002 $\left(16.7^{\circ}\right)$ and it reduces to $13.0^{\circ}$ in 2005 . The central latitude varies more than in the north, ranging from $-68^{\circ}$ in 2002 to $-76^{\circ}$ in 2004.

In Fig. 4, we note the yearly averaged P10.7 values below each dial plot. They have been retrieved by averaging the P10.7 values of the considered overpasses. Synchronously with the intensity of the density peaks, but at a lower rate, the P10.7 index decreases with the years. In fact, it reduces by a factor of 2 between 2002 and 2005 . This indicates a significant and over-proportional dependence of the density anomaly on the solar cycle.

To separate other dependences independently from the year/P10.7 distribution, the data set was sorted by season. The results are presented in Fig. 5. The format is the same as for Fig. 4. Again, the upper row displays the northern hemispheric situation, the lower row the southern hemispheric one. The expressions March equinox (ME), June solstice (JS), September equinox (SE), and December solstice (DS) refer to the day-of-year episodes 40-128, 129-220, 221-312, and 313-39, respectively. The density anomaly appears in every season. As expected, it is more distinct at northern latitudes. The peaks appear at $74^{\circ} \mathrm{cgm}$ latitude during ME and DS, but they are shifted by almost $2^{\circ}$ towards the equator during JS and SE. While the FWHM is $15.9^{\circ}$ for JS, it ranges only between $12.3^{\circ}$ and $14.0^{\circ}$ in the other seasons. The P10.7 values are more balanced between the seasons than between the years, attaining values from 123 (southern ME) up to 133 (northern DS).

The seasonal dependence of the southern hemispheric density anomaly is quite different from the northern hemispheric one: First, the amplitudes are on average smaller by $10 \%$. The smallest amplitudes occur during JS (southern winter), where the anomaly is hardly visible. Instead, we see single tracks from very active days. Second, the area of maximal density is less confined at southern latitudes, especially during ME and DS. Third, the seasonal variation of the peak location is less pronounced in the south. Owing to the fact that we took into account also active periods (when the neutral mass density is strongly enhanced) the maxima in JS and DS are governed by single tracks of storm days. They are not considered as cusp-related. Since they introduce large variations they are not further included in the interpretation.

We observe an almost permanent occurrence of the density enhancement but with a significant variability. The density bulge changes with time and season both in intensity and position. Hence, we infer a multi-parameter dependence which is considered in the following section.

\subsection{Influence of the solar wind}

The solar wind plasma and the embedded IMF feed kinetic and electric energy into the magnetosphere. We therefore investigate a possible dependence of the density anomalies on the IMF $B_{z}$ component and/or the IMF merging electric field, $E_{\text {merg }}$, as proxies for an energy input. Figure 6 presents results of a superposed epoch analysis applied to IMF $B_{z}$ and $E_{\text {merg }}$ data observed around the density peak detection time. Only the maximum $\Delta \rho$ values of each overflight, called $\Delta \rho_{\max }$, have been taken into account. Here and for all subsequent analysis we make use of air density estimates which are not normalised to $400 \mathrm{~km}$ altitude. We are not sure whether the reduction to a common height is an appropriate scaling rule for the density anomalies. To avoid smearing-out effects we focused on well-developed anomalies with amplitudes of more than $1 \times 10^{-12} \mathrm{~kg} / \mathrm{m}^{3}$, called $\Delta \rho_{\text {high }}$, leaving 5121 (2672) events in the Northern (Southern) Hemisphere. Each CHAMP overpass was checked for a sufficiently large density maximum, and the detection time was defined as the key time, $t=0$. We considered the respective solar wind parameters in time segments of $\pm 1.5 \mathrm{~h}$ around the key time, stacked the data of all overpasses and averaged the compiled index curves as running means with time steps of $10 \mathrm{~min}$. The analysis has been performed separately for the four seasons. Due to the limited number of peaks with amplitudes above $1 \times 10^{-12} \mathrm{~kg} / \mathrm{m}^{3}$ we did not take into account the southern hemispheric JS conditions in the superposed epoch analysis.

The left column of Fig. 6 displays the IMF $B_{z} / E_{\text {merg vari- }}$ ations for northern hemispheric events and the right column those for the southern events. The IMF $B_{z}$ variations show an absolute minimum shortly before the key time for every season. This feature is more prominent in the Northern Hemisphere, where it occurs between $16 \mathrm{~min}$ (December solstice) and 6 min (June solstice) before the key time. In the Southern Hemisphere, the time interval between the IMF $B_{z}$ minimum and the time of the density peak detection amounts to $15 \mathrm{~min}$ on average. Oppositely to $B_{z}$ the superposed epoch analysis for $E_{\text {merg }}$ reveals a maximum shortly before the key time. The positive excursion stretches over a longer period of $96 \mathrm{~min}$ on average (Northern Hemisphere) and $102 \mathrm{~min}$ (Southern Hemisphere). According to the mean error of the average we have to expect an uncertainty of $0.6-1.9 \%$ for $B_{z}$ and $0.1-0.2 \%$ for the $E_{\text {merg }}$. The analysis of both parameters, $B_{z}$ and $E_{\text {merg }}$, indicates that an enhanced energy input occurs shortly before the detection of a cusp density peak. In order to get a representative number for the solar wind input related to the events we calculated the mean value of $E_{\text {merg }}$ from the $40 \mathrm{~min}$ preceding the key time. With the help of this procedure each cusp density anomaly is affiliated with an $E_{\text {merg }}$ value.

In the previous section we showed that the amplitude of the density anomaly depends on the solar flux level. Here we find a relation to the solar wind input. For that reason we performed a 2-parameter analysis. Density values, $\Delta \rho_{\max }$ at 

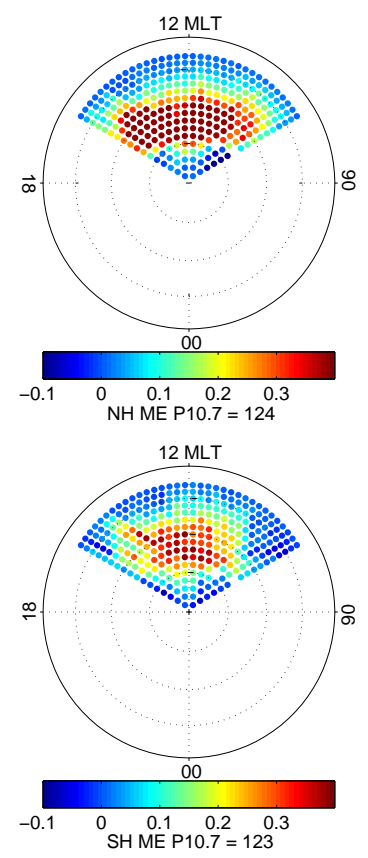
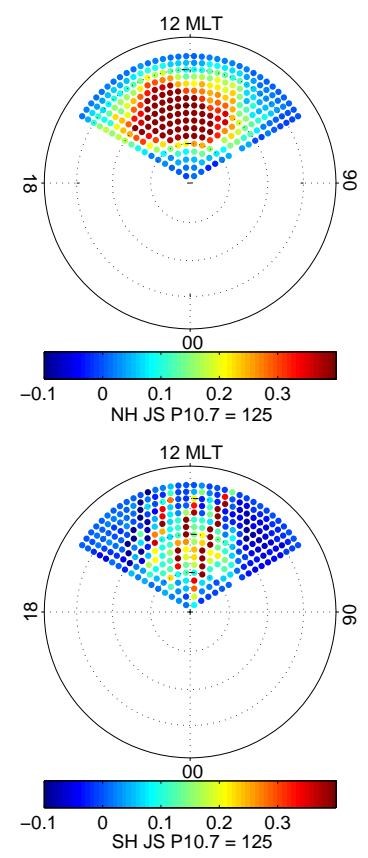
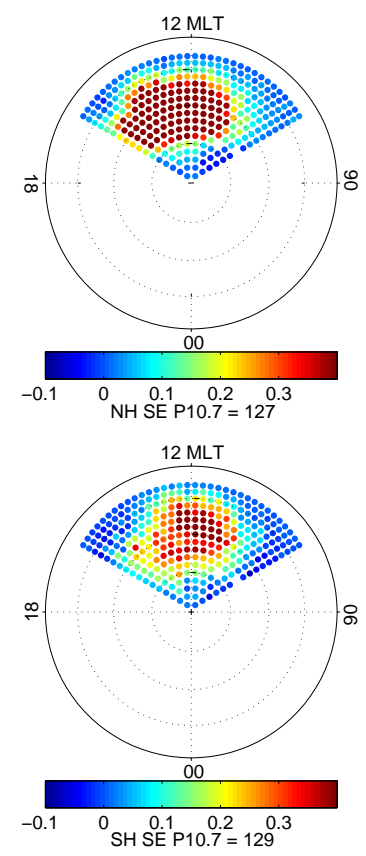
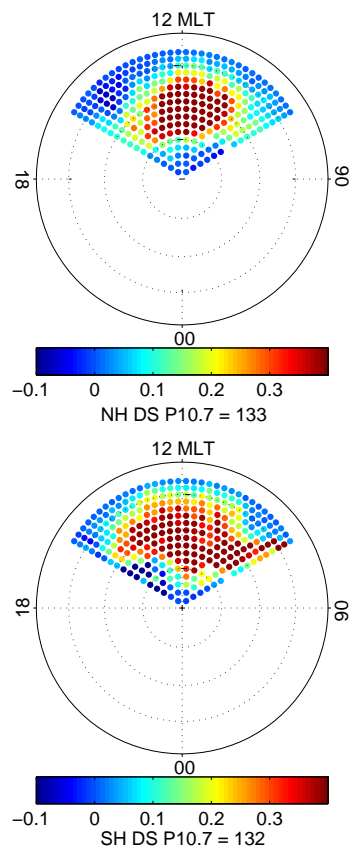

Fig. 5. Seasonal dependence of the density anomaly in the dayside polar region of the Northern (upper row) and Southern (lower row) Hemisphere for March Equinox (ME, first column), June Solstice (JS, second column), September Equinox (SE, third column), and December Solstice (DS, fourth column). Average solar flux values (P10.7) are listed below the plots.

orbital altitude, are sorted into a solar flux versus merging electric field matrix.

The results are presented in the top row of Fig. 7, where we depict the median amplitude of the density anomaly in colour, separately for the hemispheres. Here, we did not restrict the event selection to any amplitude threshold. The only criterion for the peak value to be considered is that it occurs within the latitude range $60^{\circ}$ to $80^{\circ} \mathrm{cgm}$ latitude. The most striking feature is the dominance of large density anomalies in the high P10.7/elevated $E_{\text {merg }}$ sector. This constellation occurs at northern and southern latitudes. Obviously, it requires a combination of enhanced solar flux and solar wind input for the cusp anomalies to become large. Determined quartiles are $Q .25=0.52 \times 10^{-12} \mathrm{~kg} / \mathrm{m}^{3}$ $\left(Q .25=0.27 \times 10^{-12} \mathrm{~kg} / \mathrm{m}^{3}\right)$, and $Q .75=1.30 \times 10^{-12} \mathrm{~kg} / \mathrm{m}^{3}$ $\left(Q .75=0.80 \times 10^{-12} \mathrm{~kg} / \mathrm{m}^{3}\right)$ for the Northern (Southern) Hemisphere. For geophysical conditions of P10.7<150 and $E_{\text {merg }}<1 \mathrm{mV} / \mathrm{m}$ hardly any events are detected. In both hemispheres, bins with $\mathrm{P} 10.7<150$ do not contain median densities $>2 \times 10^{-12} \mathrm{~kg} / \mathrm{m}^{3}$. There are also only less than 5 bins with density anomalies $>2 \times 10^{-12} \mathrm{~kg} / \mathrm{m}^{3}$ for $E_{\text {merg }}<1 \mathrm{mV} / \mathrm{m}$.

The same 2-D-analysis as above was performed with the relative density enhancement, $d \rho_{\text {rel }}$. We intended to determine the role of the background air density for the formation of the anomaly. The obtained results are presented in the bottom row of Fig. 7. In all cases we used density readings from the spacecraft's orbit altitude. The picture is different from the absolute amplitude of the anomaly. The dependence on solar flux level is clearly reduced. Here, the relative size of the density anomaly depends mainly on $E_{\text {merg }}$, the solar wind input. Median ratios up to 1.5 can be detected. Also for this quantity the effect is markedly stronger in the Northern Hemisphere. For merging electric fields between 1 and $2 \mathrm{mV} / \mathrm{m}$ we find relative density enhancements varying around 1.3 in the Northern Hemisphere and around 1.2 in the Southern Hemisphere. For the relative density enhancements we find quartiles of $Q_{.25}=1.13 \times 10^{-12} \mathrm{~kg} / \mathrm{m}^{3}$ $\left(Q_{.25}=1.09 \times 10^{-12} \mathrm{~kg} / \mathrm{m}^{3}\right)$, and $Q .75=1.24 \times 10^{-12} \mathrm{~kg} / \mathrm{m}^{3}$ $\left(Q .75=1.17 \times 10^{-12} \mathrm{~kg} / \mathrm{m}^{3}\right)$ for the Northern (Southern) Hemisphere, respectively. The hemispheric differences will be discussed in the next section.

\section{Discussion}

This study is focussing on thermospheric mass density anomalies occurring at auroral latitudes on the dayside. In particular from CHAMP accelerometer data there have been evidence for density bulges in the vicinity of the cusp region (e.g. Lühr et al., 2004; Liu et al., 2005; Schlegel et al., 2005). Here we make use of a CHAMP data set covering 4 years, from which we try to derive statistically relevant properties. The aim of this paper is primarily a detailed presentation of the observations. Knowing the characteristics of the density 

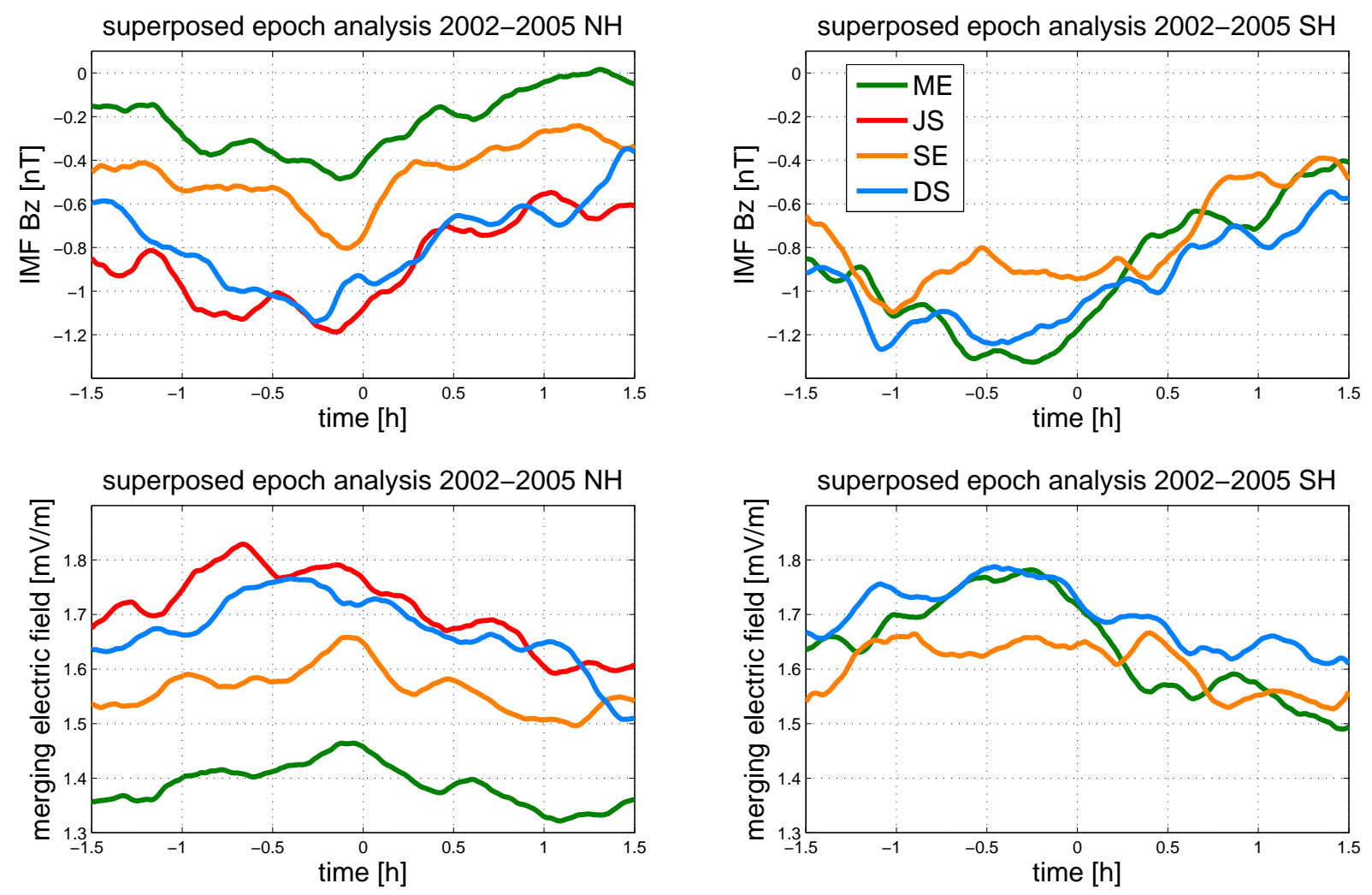

Fig. 6. Average variation of the IMF $B_{z}$ component (upper panels) and the IMF merging electric field, $E_{\text {merg }}$, (lower panels) during the $\pm 1.5 \mathrm{~h}$ around the density peak, as resulting from a superposed epoch analysis. Results from the different seasons are plotted separately. The green line indicates March equinox (ME) conditions, the red June solstice (JS), the orange September equinox (SE), and the blue line the December solstice (DS) conditions.

anomaly and its dependences on geophysical conditions will help to identify the driving mechanisms.

\subsection{Relation of the density anomaly with the cusp location}

The polar cusp is defined as the location where the magnetic field lines from the magnetopause reach the ionosphere. According to Newell and Meng (1988) the cusp is the "dayside region in which the entry of magnetosheath plasma to low altitudes is most direct. Entry into a region is considered more direct if more particles make it in (the number flux is higher) and if such particles maintain more of their original energy spectral characteristics." These authors documented its occurence between 11:00-13:00 MLT with a latitudinal width of $0.8-1.1^{\circ} \mathrm{cgm}$ latitude depending on the geomagnetic activity level. Russell (2000) found the cusp to be located between 77-90 invariant latitude for an intermediate shape of the magnetopause. Its position changes with varying magnetospheric plasma distribution, reconnection rate and reconnection location. The most precise way to describe the cusp is defining it by the energy of the incoming particles $\left(E_{e}<200 \mathrm{eV}, E_{i}<2700 \mathrm{eV}\right.$; Newell and Meng, 1988). However, we do not limit our observations to that very confined area but regard the neutral atmosphere in its wider catchment area. Therefore, talking about the cusp-related region concerning the neutral thermosphere in the following comprises also surrounding areas connected to the mantle and low latitude boundary layer (LLBL).

As can be seen in Table 1 the density anomaly is found to occur in the same MLT range as the cusp, except for the southern hemispheric DS conditions. The maxima are located at least $3^{\circ}$ to $5^{\circ}$ in latitude equatorward of the magnetospheric cusp location in the Northern Hemisphere, as quoted by Russell (2000), and $1^{\circ}$ to $11^{\circ}$ equatorward in the Southern Hemisphere. The designated cusp position of $75-76.5^{\circ} \mathrm{cgm}$ latitude for winter solstice, F10.7=165 conditions in the Sheffield High Latitude (SHL) model (Millward et al., 1999) is located even closer to our results of Table 1. It is known that the cusp moves equatorward during magnetically active periods. Within the studied period we encountered a whole range of activities. This may explain the findings in Table 1, where the FWHM stretches over a significant larger area in cgm latitudes than the highly confined definition of the magnetospheric cusp. 

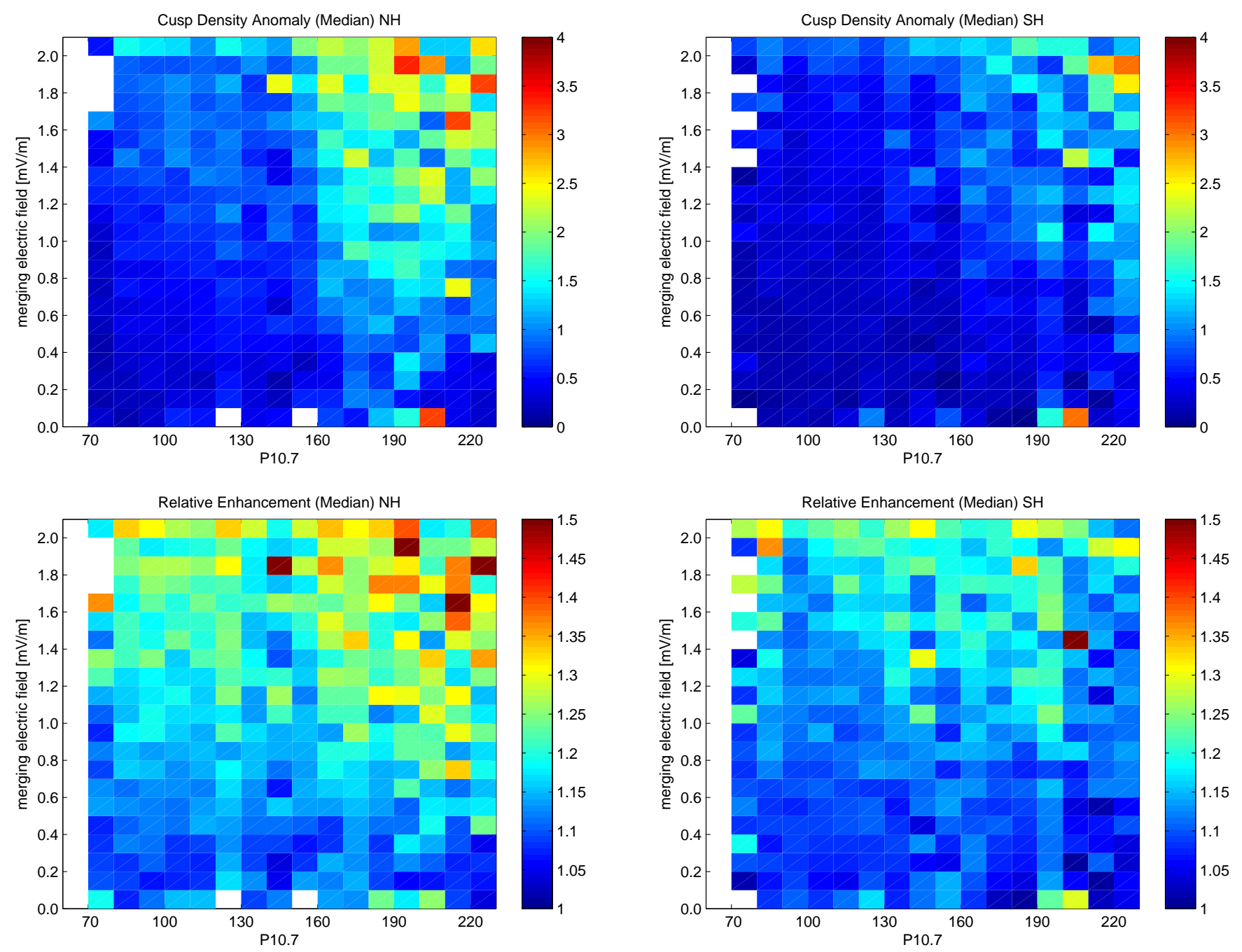

Fig. 7. A 2-D distribution of the density anomaly (in $10^{-12} \mathrm{~kg} / \mathrm{m}^{3}$, colour scale) depending on $E_{\text {merg }}$ and P10.7 for both hemispheres (upper

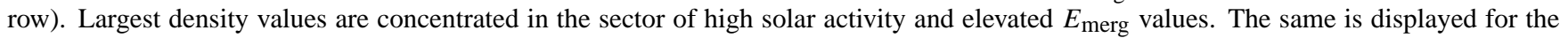
relative anomaly (lower row). Large relative enhancements occur at elevated $E_{\text {merg }}$ conditions over a wider range of P10.7 levels.

In order to check this assumption we have investigated the dependence of the anomaly location on external influences. It is well known, that the cusp moves equatorward with increasing magnetic activity. Prölss (2006) reported a close relation between the amplitude of the auroral activity, AE index, and the latitude of the cusp. Similarly, Rother et al. (2007) presented a functional relation between the latitude of their kilometre-scale field-aligned current (KS-FAC) events and the magnetic activity, $K_{p}$ index. During times of enhanced activity the cusp is displaced equatorward.

We have binned the cgm latitude of each density anomaly, $\Delta \rho_{\text {high }}$, by $K_{p}$ and determined the median latitude for each bin. In this case, the dataset was further subdivided for events in darkness $\left(\mathrm{SZA}>100^{\circ}\right)$ and in sunlight $\left(\mathrm{SZA} \leqq 100^{\circ}\right)$. Figure 8 shows the results for the Northern and Southern Hemi- sphere. In general, we see that the equatorward displacement of the density anomaly with increasing $K_{p}$ values follows the curve of the small-scale FACs reasonably well. The locations of the anomalies detected in sunlight are systematically more poleward. On average, we find a separation by about $2^{\circ}$ in latitude. This value is consistent with the reported $2^{\circ}$ equatorward shift of FAC locations around noon for events in darkness with respect to those in sunlight (Wang et al., 2005). An alternative explanation was presented by Zhou et al. (1999). They attributed the latitudinal shift of the cusp to the dipole tilt angle, not to the influence of the solar irradiation. Zhou et al. (1999) reported a relation of $1^{\circ}$ shift in latitude per $14^{\circ}$ change in tilt angle. The obtained difference of $2^{\circ}$ between the latitudes of events in darkness or sunlight corresponds to a change in tilt angle by 

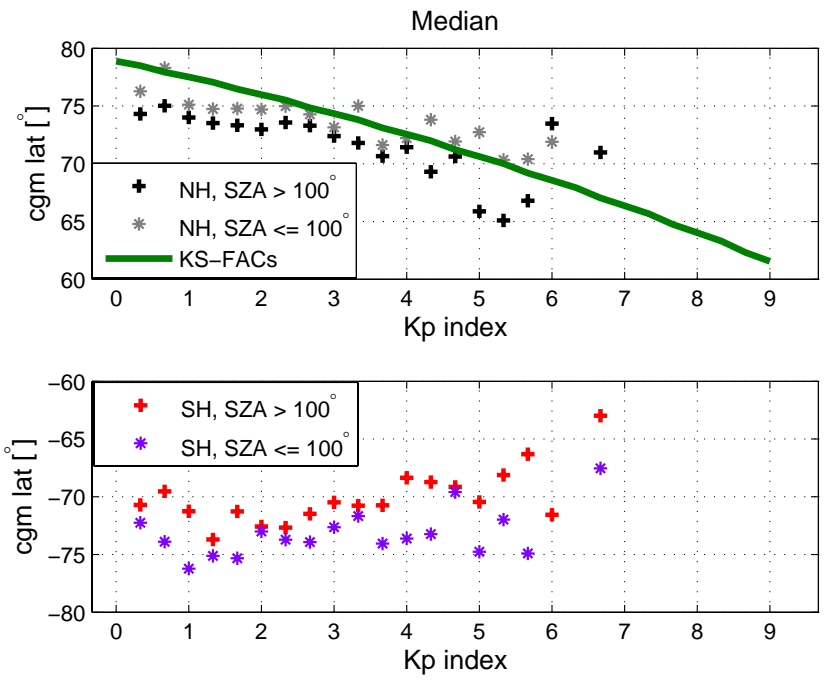

Fig. 8. Dependence of the median latitude of density anomaly peaks on the magnetic activity, $K_{p}$ index, for the Northern (top panel) and Southern (bottom panel) Hemisphere. The latitudes of the anomaly peaks are determined separately for two solar zenith angle ranges, where crosses are from dark and asterisks from sunlit conditions. For comparison, the average latitude dependence of Kilometre-Scale FACs (green line) is included.

$\pm 14^{\circ}$. This fits quite well the average shift in tilt angle between summer and winter season. We are also more in favour with the tilt angle dependence than with the influence of the sunlight. The latitude changes in the Southern Hemisphere are similar to those in the north but much more scattered.

The observed latitude variation adds confidence to our suggestion that the neutral mass density anomalies are related to the magnetospheric cusp region.

4.2 Dependence of the density anomalies on geophysical conditions

In the previous section it became clear that the strength of the anomaly is strongly dependent on preconditioning of the upper atmosphere. Very important is the intensity of the solar flux level, P10.7, on the previous day. In Fig. 4 we have seen that the amplitude of the anomaly seems to reduce faster than the solar flux level, at least within the resolvable range of our statistical analysis. The upper row of Fig. 7 seems to support these suggestions. There are practically no anomalies with amplitudes $>2 \times 10^{-12} \mathrm{~kg} / \mathrm{m}^{3}$ found for P10.7 $<130$. A somewhat different picture emerges when looking at the relative density enhancements (Fig. 7, lower row). Here, the dependence on solar flux level is much lower but still recognisable. Obviously, the absolute amplitude of the anomalies depends on both the solar flux and the background air density.
The other important controlling parameter is the solar wind input, quantified here by the merging electric field. In Fig. 7 prominent anomalies are only found for $E_{\text {merg }}>1 \mathrm{mV} / \mathrm{m}$. The resulting amplitude of the density bulge is obviously proportional to an additive effect of $E_{\text {merg }}$ and P10.7. In order to better describe the functional dependence we have performed a sensitivity analysis to characterise the weights of the two parameters:

$\Delta \rho_{\text {high }}=f\left(b E_{\text {merg }}+P 10.7+c\right)$,

where $b$, and $c$ are the parameters to be determined, and for the function, $f$, we have chosen a parabola. Based on the data presented in the top row of Fig. 7 we varied the factor $b$ in Eq. (8) until we obtained the narrowest distribution of data points around the parabolic curve. An optimal value is $b=52$ giving a reasonably narrow point distribution for observations in both hemispheres, as can be seen in Fig. 9. The weight of the merging electric field in $\mathrm{mV} / \mathrm{m}$ is thus about 50 times higher than that of the solar flux units. It is obvious from Fig. 7 that the increase in anomaly amplitude is overproportional. Furthermore, the anomalies fade away when the combined parameter drops below 100. Equation (8) provides a reasonable approximation for $\Delta \rho$ when we chose a parabolic function with the parameters $b=52$ and $c=-100$. The respective best fits for the two hemispheres are also plotted into Fig. 9. As factors for scaling the fitted curves we obtain $a_{\mathrm{NH}}=5 \times 10^{-5}$ and $a_{\mathrm{SH}}=3 \times 10^{-5}$ for the Northern and Southern Hemisphere, respectively.

It has earlier been stated that the field-aligned currents on the dayside behave like driven by a voltage source (e.g. Wang et al., 2005). The observed quadratic increase of the anomaly strength with the merging electric field is consistent with a voltage source analogue. Moreover, there seems to be a threshold of energy input that has to be surpassed before a density anomaly can form at about $400 \mathrm{~km}$ altitude. At this time we cannot offer a convincing explanation for the necessary preconditioning of the thermosphere required to start the air up-welling.

Another parameter that has been considered is the solar zenith angle (SZA). It is known, that the amount of sunlight reaching the polar ionosphere is one of the prime controlling factors for the conductivity on the dayside. Furthermore, it has been shown by Wang et al. (2005) that the intensity of field-aligned currents and electrojets is strongly dependent on SZA in the local time sector around noon. If we regard Joule heating as a cause for the air up-welling in the thermospheric dayside high latitudes, then the conductivity should be a relevant parameter.

A dependence on SZA is expected to be evident rather clearly in the seasonal differences. Figure 5 shows the intensity and distribution of the anomaly separated by season. Interestingly, we obtain weakest anomalies in both hemispheres during June solstice months. It is thus difficult to explain that the observed seasonal variations are caused by 

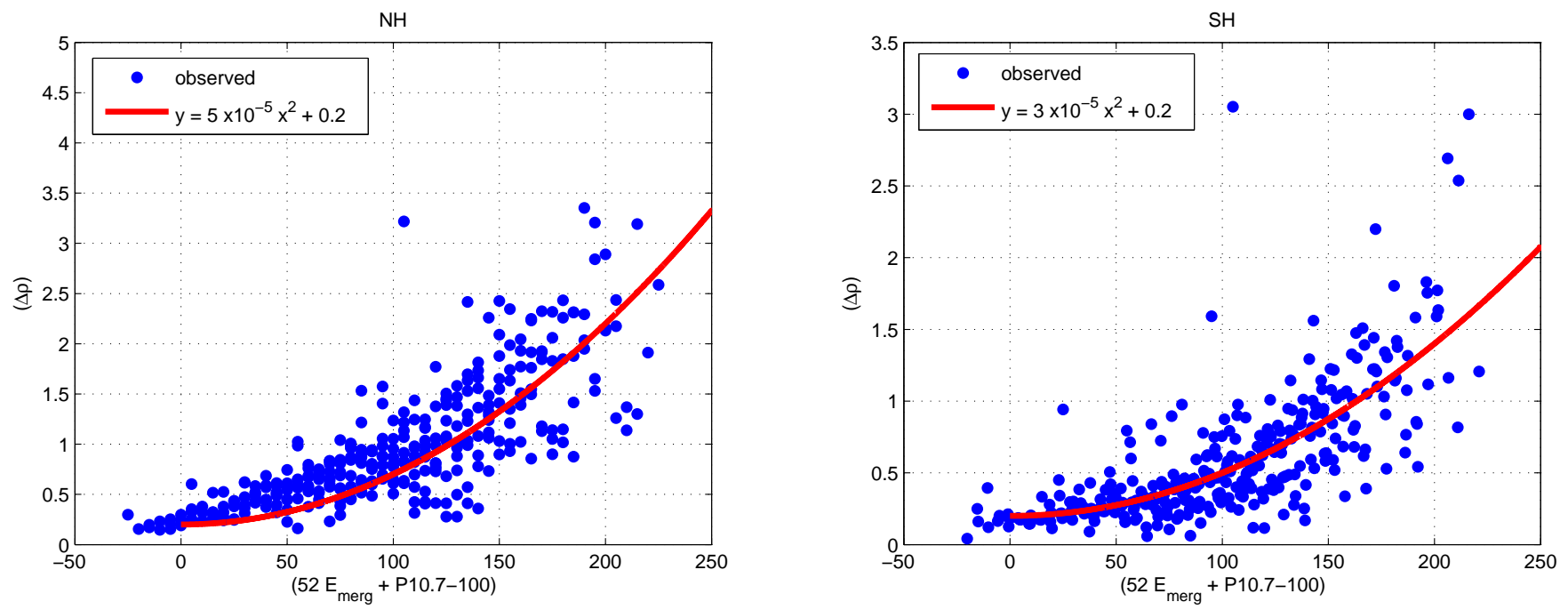

Fig. 9. Dependence of the density anomaly on the optimal linear combination of the controlling parameters. There seems to be a quadratic relation between the anomaly strength and the parameters. The red curve represents the parabolic best fit to the observations.
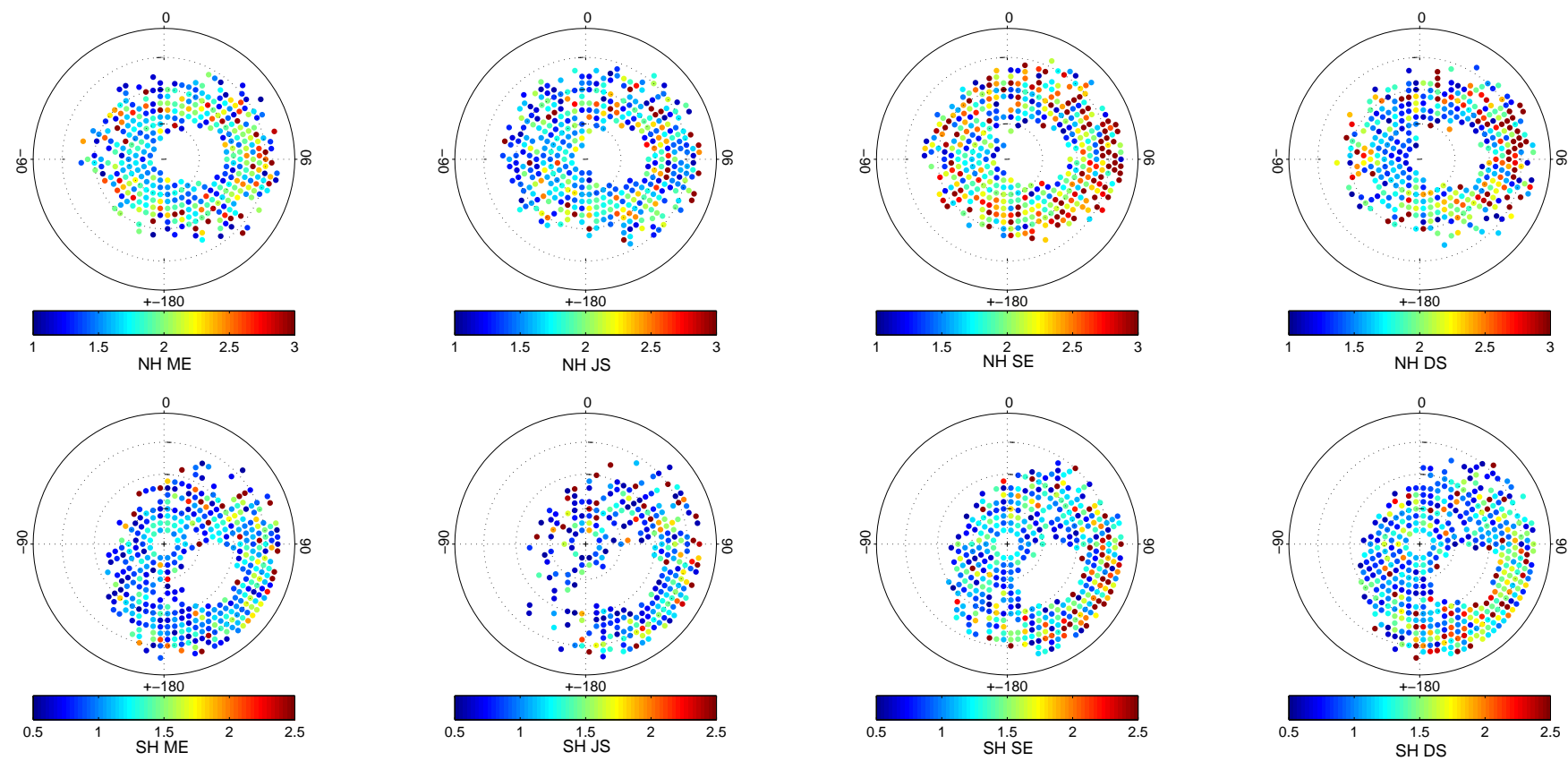

Fig. 10. The location of the density anomaly peaks in geographic coordinates separately for both hemispheres and the four seasons. Stronger anomalies are more common at lower latitudes.

changes in photoionisation. There seems to be another annual variation of the ionosphere-thermosphere system with strong control on the formation of the cusp-related anomalies. In order to separate between this effect and the SZA dependence we have plotted the location and amplitude of the density anomalies on geographic coordinates in Fig. 10. As expected, the anomalies are confined to auroral latitudes, leaving a void in the polar cap. The blank circle at the pole is the region not sampled by CHAMP. In this coordinate frame, the SZA changes almost linearly with latitude. We may thus identify SZA dependences in Fig. 10. Although large amplitudes (red dots) can be found at all longitudes, there is a somewhat higher concentration at longitudes where the cusprelated area reaches down to lower geographic latitudes. This relation is more evident in the Southern Hemisphere, where we have a larger distance between geographic and geomagnetic poles. 


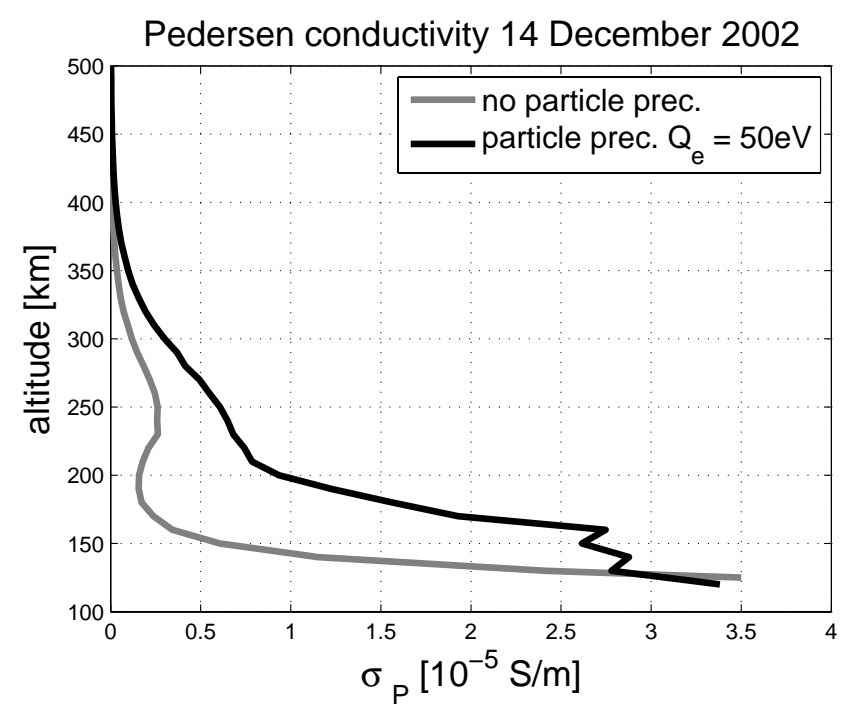

Fig. 11. Height profile of the Pedersen conductivities according to the electron concentration as derived from model runs of Millward et al. (1999) for 14 December 2002, 09:30 UT. This day is characterised by elevated solar activity $(\mathrm{F} 10.7=167)$ and moderate geomagnetic activity $\left(K_{p}=2.7\right)$. Considered are the cases of absence of precipitating particles (grey) and presence of precipitation (black).

From this behaviour we may conclude that the amount of solar irradiation has a certain but limited influence on the amplitude of the cusp anomaly. The main seasonal differences can, however, not be explained by the SZA.

An important source of ionospheric conductivity in the dark auroral region is particle precipitation. Here we give an order of magnitude estimate based on model predictions because we are not taking actual particle measurements into account in this study. With the help of the CTIP model Millward et al. (1999) have modelled the effect of particle precipitation on the electron density distribution using typical cusp conditions (Maxwellian energy distribution of $50 \mathrm{eV}$ electrons and $500 \mathrm{eV}$ ions). From their electron density profiles (their Figs. 8 and 9) we calculated the Pedersen conductivity adopting the geophysical conditions quoted there $\left(K_{p}=2.7\right.$, F10.7=167). In Fig. 11 we have plotted the resulting height profiles of the Pedersen conductivity for particle precipitation switched on and off. It is quite evident that the conductivity is significantly enhanced, in particluar in the $\mathrm{F}$ region above $150 \mathrm{~km}$. This would cause that the altitude of effective Joule heating is lifted up. With that the heating rate per particle increases and a larger temperature is achieved in the heated region. A more detailed investigation of the role of precipitating particles should be part of a subsequent study.

Particle precipitation in the cusp is generally associated with reconnection at the magnetopause. The merging electric field, $E_{\text {merg }}$, has been identified as a measure for the reconnection rate. For this reason $E_{\text {merg }}$ may have a two-fold
Table 2. Average ambient mass density in the cusp region at a flux level $\mathrm{P} 10.7=150$, separated by season and hemisphere. The data are adopted from Liu et al. (2007).

\begin{tabular}{lcc}
\hline season & $\begin{array}{c}\mathrm{NH} \\
\text { Density } \\
{\left[10^{-12} \mathrm{~kg} / \mathrm{m}^{3}\right]}\end{array}$ & $\begin{array}{c}\mathrm{SH} \\
\text { Density } \\
{\left[10^{-12} \mathrm{~kg} / \mathrm{m}^{3}\right]}\end{array}$ \\
\hline March equinox & $7.02 \pm 0.20$ & $6.75 \pm 0.35$ \\
June solstice & $5.81 \pm 0.25$ & $4.10 \pm 0.30$ \\
September equinox & $5.92 \pm 0.20$ & $6.15 \pm 0.35$ \\
December solstice & $5.02 \pm 0.25$ & $6.40 \pm 0.25$ \\
\hline
\end{tabular}

significance for the cusp-related density anomaly (1) it reflects the electric field driving the ionospheric currents and (2) indicates the rate at which particle precipitation events occur and thus enhance the conductivity. Dedicated studies are required to determine the role of particle precipitation for the cusp-related density anomaly quantitatively.

The remaining question is, which atmospheric parameter is causing the global annual variation of the cusp-related density anomaly? To answer this question we first took a look at the ambient air density prevailing during the anomaly events. In a recent paper, Liu et al. (2007) have studied the tempo$\mathrm{ral} / \mathrm{spatial}$ variation of the thermospheric density on the dayside for the same four years. In their Fig. 4 they present latitudinal profiles of the thermospheric density separately for each season. The difference in the December-June asymmetry between the two polar regions is quite evident in their figure. We have listed their reported average mass density values at $\pm 60^{\circ} \mathrm{cgm}$ latitude separately for all seasons in Table 2. Prevailing ambient conditions for these numbers are $\mathrm{P} 10.7=150, K_{p} \leq 2$ and an altitude of $400 \mathrm{~km}$. These seasonal variations of the thermospheric density match rather well the amplitude variation of the cusp-related anomaly, as shown in Fig. 5. An annual variation of the thermospheric density has earlier been deduced from satellite orbit analysis. For example, Boulton (1985) reported an air density in the altitude range $240-470 \mathrm{~km}$ that is 1.3 times larger in December than in June. Up to date there is no consensus on the mechanism causing this major annual variation. As a viable candidate an atmospheric composition change is discussed (e.g. Fuller-Rowell, 1997).

The combination of the annual variation with the seasonal variation of the solar insolation produces the strong hemispheric asymmetry during solstices. In the Northern Hemisphere, these two effects are out of phase. The global reduction in air density during June solstice is locally compensated by continuous sunshine. In the Southern Hemisphere both phenomena vary in phase thus causing large differences between solstice seasons (cf. Table 2). Unfortunately, CHAMP does not provide any information on composition. Therefore, we cannot contribute to explaining the annual variation of the thermospheric density. With respect to this study we may 
recall that an enhanced ambient air density is in favour with the formation of cusp anomalies. The much reduced thermospheric density in the southern polar region during June solstice is suggested to be the cause for the infrequent occurrence of density anomalies in that season.

\section{Conclusions}

Here we have presented the first systematic survey of the thermospheric mass density in the high-latitude dayside thermosphere. Based on 4 years (2002-2005) of CHAMP air drag measurements climatological properties of the density anomaly could be derived from a statistical study.

The anomaly is rather confined to the ionospheric location of the cusp. For that reason, we relate it primarily to energy input from the magnetosphere. According to model calculations (Demars and Schunk, 2007) a strong air up-welling is associated with the phenomenon. The up-welling air is suggested to diverge at a certain altitude equatorward and poleward. Therefore, we regard the cusp-related anomalies as an important source for the upper thermospheric density distribution on the dayside. One may even speculate that they play a role in the formation of the mid-latitude density bulges (Liu et al., 2005, 2007).

The amplitude of the anomaly depends on many factors. Continuous observations over a sufficiently long period are needed for delineating all the different influences. The controlling parameters we identified are

1. Solar wind input: The merging electric field is enhanced for about an hour before the detection of the anomaly. This observed time delay may be explained by two effects, the propagation of the density front from the lower thermosphere to $400 \mathrm{~km}$ altitude and the infrequent sampling of the cusp (once per orbit). The increase in density is proportional to the square of the merging electric field.

2. Solar EUV flux: The amplitude of the anomalies depends strongly on the solar flux index P10.7. It seems that for a certain solar flux level, e.g. P10.7<100, no clear anomalies form anymore.

3. Background thermospheric density: An elevated air density clearly favours the formation of cusp density bulges. This effect is highly correlated with the conclusion above, but the solar flux to density ratio depends on season and hemisphere. We conclude that the ambient air density is the primary factor influencing the anomaly.

4. Hemispheric differences: The sensitivity to external forcing is different for the two hemispheres. According to the results in Fig. 9 the anomalies in the Northern Hemisphere are larger by a factor of 1.35 than in the
Southern for the same $E_{\text {merg }}$ and P10.7 inputs. We regard this partly to the larger offset between geographic and geomagnetic poles in the south.

5. Solar zenith angle: Only a weak dependence of the anomalies on the solar zenith angle could be detected. This observed impact might even be biased by the correlation between the SZA and the ambient density. We may therefore conclude that the SZA-related change in ionospheric conductivity does not play an important role in the anomaly formation An impact of particle precipitation on conductivity has been investigated qualitatively. No important effect was deduced.

These listed characteristics and dependences can be regarded as important constrains for the modelling of the cusp anomaly, and they will help to identify the processes responsible for the local air up-welling. This will be the topic of a follow-on paper.

Acknowledgements. We thank W. Köhler for pre-processing the accelerometer data and T. Fuller-Rowell for fruitful discussions. We are obliged to the ACE MAG and SWEPAM instrument teams and the ACE Science Center for providing the ACE data. The operational support of the CHAMP mission by the German Aerospace Centre (DLR) and the financial support for the data processing by the Federal Ministry of Education and Research (BMBF), as part of the Geotechnology Programme, are gratefully acknowledged. S. R. was supported by the Deutsche Forschungsgemeinschaft DFG through the DFG Priority Programme "CAWSES", SPP 1176.

Topical Editor M. Pinnock thanks A. Rodger and another anonymous referee for their help in evaluating this paper.

\section{References}

Appleton, E. V. and Ingram, L. J.: Magnetic storms and upper atmospheric ionisation, Nature, 136, 548-549, doi:10.1038/136548b0, 1935.

Boulton, W. J.: The determination and analysis of the orbit of Nimbus 1 rocket, 1964-52b: The semi-annual variation in air density from June 1968 to August 1974, Planet. Space Sci., 33, 14171431, 1985.

Bruce, R. W.: Upper atmospheric density determination from LOGACS, in The Low-G Accelerometer Calibration System, Vol. II, Rept. No. TR-0074(4260-10), Vol. II, The Aerospace Corp., El Segundo, CA, 1-1-1-43, 1973.

Bruinsma, S. D., Tamagnan, D., and Biancale, R.: Atmospheric densities derived from CHAMP/STAR accelerometer observations, Planet. Space Sci., 52, 297-312, 2004.

Demars, H. G. and Schunk, R. W.: Thermospheric response to ion heating in the dayside cusp, J. Atmos. Sol.-Terr. Phys., 69, 649660, 2007

Frank, L. A.: Plasma in the Earth's polar magnetosphere, J. Geophys. Res., 76, 5202-5219, 1971.

Fuller-Rowell, T. J., Codrescu, M. V., Fejer, B. G., Borer, W., Marcos, F., and Anderson, D. N.: Dynamics of the low-latitude thermosphere: quiet and disturbed conditions, J. Atmos. Terr. Phys., 61, 1533-1540, 1997. 
Guo, J., Wan, W., Forbes, J. M., Sutton, E., Nerem R. S., Woods, T. N., Bruinsma, S., and Liu, L.: Effects of solar variability on thermosphere density from CHAMP accelerometer data, J. Geophys. Res., 112, A10308, doi:10.1029/2007JA12409, 2007.

Heikkila, W. J. and Winningham, J. D.: Penetration of magnetosheath plasma to lower altitudes through the dayside magnetospheric cusps, J. Geophys. Res., 76, 883-891, 1971.

Jacchia, L. G.: Solar effects on the acceleration of artificial satellites, Smithsonian Astrophysical Observatory, Special Report No. 29, Cambridge, MA, 1959.

Jacchia, L. G.: A working model for the upper atmosphere, Nature, 192, 1147-1148, 1961.

Jacchia, L. G.: Static diffusion models of the upper atmosphere, Smithsonian Astrophysical Observatory, Special Report No. 170, Cambridge, MA, 1964.

Jacchia, L. G. and Slowey, J.: Atmospheric heating in auroral zones, Smithsonian Astrophysical Observatory, Special Report No. 136, Cambridge, MA, 1963.

Jacchia, L. G. and Slowey, J.: Atmospheric heating in the auroral zones: a priliminary analysis of the atmospheric drag of the Injune 3 satellite, J. Geophys. Res., 69(5), 905-910, 1964.

Jacobs, R. L.: Atmospheric density derived from the drag of eleven low altitude satellites, J. Geophys. Res., 72(5), 1571-1581, 1967.

Kan, J. R. and Lee, L. C.: Energy coupling function and solar windmagnetosphere dynamo, Geophys. Res. Lett., 6, 577-580, 1979.

Lathuillère, C. and Brekke, A.: Ion compositions in the auroral ionosphere as observed by EISCAT, Ann. Geophys., 3, 557-567, 1985, http://www.ann-geophys.net/3/557/1985/.

Liu H., Lühr, H., Henize, V., and Köhler, W.: Global distribution of the thermospheric total mass density derived from CHAMP. J. Geophys. Res., 110, A04301, doi:10.1029/2004JA010741, 2005.

Liu, H., Lühr, H., Watanabe, S., Köhler, W., Henize, V., and Visser, P.: Zonal wind in the equatorial upper thermosphere: decomposing the solar flux, geomagnetic activity, and seasonal dependences, J. Geophys. Res., 111, A07307, doi:10.1029/2005JA011415, 2006.

Liu, H., Lühr, H., and Watanabe, S.: Climatology of the equatorial thermospheric mass density anomaly, J. Geophys. Res., 112, A05305, doi:10.1029/2006JA012199, 2007.

Liu, L., Wan, W., Ning, B., Pirog, O. M., and Kurkin, V. I.: Solar activity variation of the ionospheric peak electron density, J. Geophys. Res., 111, A08304, doi:10.1029/2006JA011598, 2006.

Lühr, H., Rentz, S., Ritter, P., Liu, H., and Häusler, K.: Average thermospheric wind patterns over the polar regions, as observed by CHAMP, Ann. Geophys., 25, 1093-1101, 2007, http://www.ann-geophys.net/25/1093/2007/.

Lühr, H., Rother, M., Köhler, W., Ritter, P., and Grunwaldt, L.: Thermospheric up-welling in the cusp region: evidence from CHAMP observations, Geophys. Res. Lett., 31, L06805, doi:10.1029/2003GL019314, 2004.

Menvielle, M., Lathuillère, C., Bruinsma, S., and Viereck, R.: A new method for studying the thermospheric density variability derived from CHAMP/STAR accelerometer data for magnetically active conditions, Ann. Geophys., 25, 1949-1958, 2007, http://www.ann-geophys.net/25/1949/2007/.

Millward, G. H.,Moffett, R. J., Balmforth, H. F., and Rodger, A. S.: Modeling the ionospheric effects of ion and electron precipitation in the cusp, J. Geophys. Res., 104(A11), 24 603-24612, 1999.
Mitra, S. K.: The upper atmosphere, The Royal Asiatic Society of Bengal, Calcutta, 91-92, 131-143 and 417-418, 1947.

Moe, K. and Moe, M. M.: A dynamic model of the neutral thermosphere, Rep. MDLG5891, Mc Donnell Douglas Astronaut. Co., Huntington Beach, Calif., September 1975.

Moe, K., Moe, M. M., Carter, V. L., and Ruggera Jr., M. B.: The correlation of thermospheric densities with charged particle precipitation through the magnetospheric cleft, J. Geophys. Res., 82(22), 3304-3306, 1977.

Newell, P. T. and Meng, C. I.: Mapping the dayside ionosphere to the magnetosphere according to particle precipitation characteristics Geophys. Res. Lett., 19(6), 609-612, 1992.

Newell, P. T. and Meng, C. I.: The cusp and the cleft/boundary layer: low-altitude identification and statistical local time variation, J. Geophys. Res., 93(A12), 14 549-14 556, 1988.

Nicolet, M.: Properties and constitution of the earth's upper atmosphere, in: Physics of the Upper Atmosphere, edited by: Ratcliffe, J. A., Academic Press, NY and London, 17-71, 1960.

Olson, W. P.: Corpuscular radiation as an upper atmospheric energy source, in Space Research XII, b, Akademie-Verlag, Berlin, 1007-1013, 1972.

Pätzold, H.-K.: Model for the variability of the terrestrial atmosphere after satellite acceleration, U. of Cologne (1962); reprinted in Flight Performance Handbook for Orbital Operations, edited by: Wolverton, R. W., John Wiley, NY and London, 2-430-2-356, 1963

Picone, J. M., Hedin, A. E., Drob, D. P., and Aikin, A. C.: NRLMSISE-00 empirical model of the atmosphere: Statistical comparisons and scientific issues, J. Geophys. Res., 107(A12), 1468, doi:10.1029/2002JA009430, 2002.

Prölss, G. W.: Electron temperature enhancement beneath the magnetospheric cusp, J. Geophys. Res., 111, A07304, doi:10.1029/2006JA011618, 2006.

Reigber, C., Lühr, H., and Schwintzer, P.: CHAMP mission status, Adv. Space Res., 30, 129-134, 2002.

Richards, P. G., Fennelly, J. A., and Torr, D. G.: EUVAC: A solar EUV flux model for aeronomic calculations, J. Geophys. Res., 99, 8981-8992, 1994.

Richmond, A. D.: Ionospheric electrodynamics using magnetic Apex coordinates, J. Geomagn. Geoelectr., 47, 191-212, 1995.

Ritter, P., Lühr, H., Maus, S., and Viljanen, A.: High latitude ionospheric currents during very quiet times: their characteristics and predictabilities, Ann. Geophys., 22, 2001-2014, 2004, http://www.ann-geophys.net/22/2001/2004/.

Rother, M., Schlegel, K., and Lühr, H.: CHAMP observation of intense kilometer-scale field-aligned currents, evidence for an ionospheric Alfvén resonator, Ann. Geophys., 25, 1603-1615, 2007, http://www.ann-geophys.net/25/1603/2007/.

Russell, C. T.: The polar cusp, Adv. Space Res., 25(7/8), 1413 1424, 2000.

Russell, C. T., Chappell, C. R., Montgomery, M. D., Neugebauer, M., and Scarf, F. L.: OGO-5 observations of the polar cusp on Nov 1, 1968, J. Geophys. Res., 76(28), 6743-6764, 1971.

Schlegel, K.: Auroral E-region conductivities during solar minimum derived from EISCAT data, Ann. Geophys., 6, 129-138, 1988, http://www.ann-geophys.net/6/129/1988/.

Schlegel, K., Lühr, H., St.-Maurice, J.-P., Crowley, G., and Hackert, C.: Thermospheric density structures over the polar regions observed with CHAMP, Ann. Geophys., 23, 1659-1672, 2005, 
http://www.ann-geophys.net/23/1659/2005/.

Stubbe, P.: The ionosphere as a plasma laboratory, in: Modern Ionospheric Science, edited by: Kohl, H., Rüster, R., and Schlegel, K., Europaen Geophysical Society, Katlenburg-Lindau, FRG, 274-321, 1996.

Sutton, E. K., Forbes, J. M., and Nerem, R. S.: Global thermospheric neutral density and wind response to the severe 2003 geomagnetic storms from CHAMP accelerometer data, J. Geophys. Res., 110, A09S40, doi:10.1029/2004JA010985, 2005.

Vennerstrøm, S., Moretto, T., Olsen, N., Friis-Christensen, E., Stampe, A. M., and Watermann, J. F.: Field-aligned currents in the dayside cusp and polar cap region during northward IMF, J. Geophys. Res., 107(A8), 1188, doi:10.1029/2001JA009162, 2002.
Vogt, J.: Alfvén wave coupling in the auroral current circuit, Surv. Geophys., 23, 335-377, 2002.

Wang, H., Lühr, H., and Ma, S. Y.: Solar zenith angle and merging electric field control of field-aligned currents: a statistical study of the southern hemisphere, J. Geophys. Res., 110, A03306, doi:10.1029/2004JA010530, 2005.

Yordanova, E., Sundkvist, D., Buchert, S. C., André, M., Ogawa, Y., Morooka, M., Margithu, O., Amm, O., Fazakerley, A. N., and Réme, H.: Energy input from the exterior cusp into the ionosphere: correlated ground-based and satellite observations, Geophys. Res. Lett., 34, L04102, doi:10.1029/2006GL028617, 2007.

Zhou, X.-W., Russell, C. T., Le, G., Fuselier, S. A., and Scudder, J. D.: The polar cusp location and its dependence on dipole tilt, Geophys. Res. Lett., 26(3), 429-432, 1999. 